\title{
DETERMINACIÓN DEL CONTENIDO DE GLIADINAS EN ALIMENTOS ELABORADOS CON AMARANTO, QUÍNOA Y/O CHÍA
}

\author{
GLIADINS DETERMINATION IN FOOD \\ ELABORATED WITH AMARANTH, QUÍNOA AND/OR CHÍA
}

\author{
Laura Beatriz López, Luis Marcelo Dyner, \\ Silvina Mariela Vidueiros,Anabel Pallaro, Mirta Eva Valencia
}

Cátedras de Bromatología y Nutrición. Facultad de Farmacia y Bioquímica.

Universidad de Buenos Aires. Buenos Aires. Argentina.

\begin{abstract}
Amaranth, quínoa and chía are naturally gluten-free products that may be used in a celiac diet. An ELISA, using R-Biopharm RIDASCREEN gliadin, was used to determine a possible cross contamination with gliadins. Thirty seven samples of foods with these ingredients were analyzed. Nine samples had levels higher than $20 \mathrm{mg} / \mathrm{Kg}$, the maximum gluten level established by Codex Alimentarius: three of them were cereal bars with the inscription isin TACCî and/or ìwithout glutenî, two were cereal bars without inscriptions about gluten content, one was a mixture of ground seeds, others were pop amaranth and quinoa crops (sold at retail) and the last was an amaranth flour which was labeled ifor celiac patientsî. Twenty-eight remaining samples had a gluten content below $20 \mathrm{mg} / \mathrm{Kg}$. Foods elaborated with amaranth, quinoa and/or chía are suitable for celiac patients. However, the manufacturers must apply good manufacturing practices in all the different steps in gluten-free foodstuff production and celiac patients should not buy these products when they are sold at retail, because of possible cross contamination that can occur at the stores.
\end{abstract}

Key words: Amaranth; quinoa; chía; gliadin; celiac disease.

Este trabajo fue recibido el 23 de Marzo de 2009 y aceptado para ser publicado el 10 de Diciembre de 2009.

\section{INTRODUCCIÓN}

La celiaquía o enfermedad celíaca es la intolerancia permanente a un conjunto de proteínas denominadas prolaminas, presentes en el trigo, la avena, la cebada y el centeno (TACC) y en productos derivados de estos cuatro cereales. Las prolaminas reciben distintos nombres de acuerdo al cereal de procedencia: trigo: gliadina, avena: avenina, cebada: hordeína y centeno: secalina. El gluten de los cereales mencionados es la forma más conocida de presentación de las prolaminas tóxicas para los celíacos. La gliadina es las más utilizada a nivel industrial y constituye el mayor problema ya que en muchos alimentos la harina de trigo está presente como ingrediente principal o como aditivo y en otros está presente por posibles contaminaciones cruzadas que pueden darse a nivel industrial o en la comercialización de los alimentos.

Las prolaminas tóxicas para los enfermos celíacos afectan directamente al intestino delgado, en el cual se produce una severa lesión con atrofia de las vellosidades intestinales. Esto conlleva una inadecuada absorción de los nutrientes de los alimentos (1-3).

El único tratamiento para revertir los síntomas de la enfermedad celíaca es una dieta estricta y permanente libre de gluten de TACC (1-3). Por este motivo es de suma importancia para los pacientes con esta patología disponer de alimentos libres de gluten que resulten seguros para su dieta, es decir alimentos que no sólo no contengan proteínas de cereales TACC por su composición si no que además no hayan sufrido una contaminación cruzada con cereales TACC en ninguna de las etapas, desde su proceso de elaboración hasta su comercialización final.

El Codex Alimentarius (4) considera exentos de gluten a los alimentos que están constituidos por, o son elaborados únicamente con, uno o más ingredientes 
que no contienen trigo (es decir, todas las especies de Triticum, como el trigo duro, la espelta y el kamut), centeno, cebada, avena o sus variedades híbridas, y cuyo contenido de gluten no sobrepasa los $20 \mathrm{mg} / \mathrm{kg}$ en total, medido en los alimentos tal como se venden o distribuyen al consumidor. Establece además que la determinación de la cantidad de gluten presente en los alimentos e ingredientes deberá basarse en un método inmunológico o en otro método que ofrezca como mínimo la misma sensibilidad y especificidad (4).

El amaranto (Amaranthus caudatus L), la quínoa (Chenopodium quinoa W) y la chía (Salvia hispanica L) constituyen antiguos cultivos americanos que actualmente se han revalorizado por su elevado potencial en la alimentación (5). Por ser naturalmente libres de gluten, estos alimentos pueden ser incorporados en la dieta celíaca (3). Sin embargo, en su manipulación puede ocurrir contaminación cruzada con cereales no permitidos.

Con el objeto de evaluar la existencia de una posible contaminación cruzada, se realizó la detección y cuantificación de gliadinas con un enzimoinmunoensayo (ELISA), en treinta y siete productos (comerciales o elaborados en planta piloto) que contienen como ingredientes amaranto, quínoa y/o chía.

\section{MATERIALES Y MÉTODOS}

Alimentos: se analizaron treinta y siete productos que contenían en forma exclusiva o combinada amaranto (Amaranthus caudatus L), quínoa (Chenopodium quinoa W) y/o chía (Salvia hispanica L). Los productos analizados correspondían a dos harinas (1 y 2), nueve semillas (3-11), tres granos inflados o popeados (12-14), tres extrudados (15-17), dos hojuelas (18 y 19), tres granolas (20-22), una hamburguesa (23), tres galletas (24-26) y once barras (27-37).

Ocho de las muestras presentaban alguna declaración relacionada con la ausencia de gluten y/o cereales TACC o apta para celíacos $(2,26-28,30,31,36,37)$.

A continuación se describen las características principales de las muestras analizadas y los ingredientes utilizados/declarados en cada uno de ellos.

1: Harina integral de amaranto, obtenida en el Instituto de Tecnología de Alimentos (ITA), ciudad de Santa $\mathrm{Fe}$, provincia de Santa $\mathrm{Fe}$, Argentina. No es un producto de origen comercial.

2: Harina de amaranto envasada, comprada en un almacén de alimentos dietéticos de la Ciudad Autónoma de Buenos Aires, Argentina. Declara "apto para celíacos".

3: Semillas de amaranto, compradas al detalle en un almacén de alimentos dietéticos de la Ciudad Au- tónoma de Buenos Aires, Argentina.

4: Semillas de amaranto compradas al detalle en un almacén de alimentos dietéticos de la Ciudad Autónoma de Buenos Aires, Argentina.

5, 6 y 7: Semillas de quínoa compradas al detalle en tres almacenes de alimentos dietéticos distintos de la Ciudad Autónoma de Buenos Aires, Argentina.

8: Semillas de quínoa compradas al detalle en un mercado de la Ciudad de Salta, provincia de Salta, Argentina.

9: Semillas de quínoa envasadas, compradas en un almacén de alimentos dietéticos de la Ciudad Autónoma de Buenos Aires, Argentina

10: Semillas de chía envasadas, compradas en un almacén de alimentos dietéticos de la Ciudad Autónoma de Buenos Aires, Argentina.

11: "Mix de Semillas molidas". Ingredientes: semillas de girasol molidas, semillas de amaranto molidas, semillas de uva molidas, semillas de avena molidas, alfalfa molida. El producto fue adquirido en un almacén de alimentos dietéticos de la Ciudad Autónoma de Buenos Aires, Argentina.

12: Granos de amaranto inflados, envasados. El producto fue comprado en un almacén de alimentos dietéticos de la Ciudad Autónoma de Buenos Aires, Argentina.

13: Amaranto popeado comprado al detalle en un almacén de alimentos dietéticos de la Ciudad Autónoma de Buenos Aires, Argentina.

14: Amaranto popeado comprado al detalle en un almacén de alimentos dietéticos de la Ciudad Autónoma de Buenos Aires, Argentina.

15: Extrudado de arroz y harina integral de amaranto, elaborado en planta piloto, ITA, ciudad de Santa Fe, provincia de Santa Fe, Argentina. No es un producto de origen comercial (corresponde a un desarrollo del ITA).

16: Extrudado de maíz y harina integral de amaranto, elaborado en planta piloto, ITA, ciudad de Santa Fe, provincia de Santa Fe, Argentina. No es un producto de origen comercial (corresponde a un desarrollo del ITA).

17: Amaranto extrudado, elaborado en planta piloto, ITA, ciudad de Santa Fe, provincia de Santa Fe, Argentina. No es un producto de origen comercial (corresponde a un desarrollo del ITA).

18: Hojuelas de quínoa envasadas. El producto fue comprado en un almacén de alimentos dietéticos de la Ciudad Autónoma de Buenos Aires, Argentina.

19: Hojuelas de amaranto envasadas. El producto fue comprado en un almacén de alimentos dietéticos de la Ciudad Autónoma de Buenos Aires, Argentina. 
20: Granola de hojuelas de quínoa sin azúcar ni edulcorantes artificiales. Ingredientes: hojuelas de quínoa tostadas libres de agroquímicos, amaranto inflado, castañas de cajú, semillas de girasol orgánico, semillas de chía molidas, harina de algarroba, yerba dulce molida, canela, coriandro, jengibre. El producto fue adquirido en un almacén de alimentos dietéticos de la Ciudad Autónoma de Buenos Aires, Argentina.

21: Granola de hojuelas de quínoa y semillas de chía. Ingredientes: hojuelas de quínoa tostadas, nueces libres de agroquímicos, semillas de chía molidas, azúcar integral orgánica tipo mascabo, coco rallado y canela. El producto fue comprado en un almacén de alimentos dietéticos de la Ciudad Autónoma de Buenos Aires, Argentina.

22: Granola de hojuelas de quínoa y semillas de chía (con pasas de uva). Ingredientes: hojuelas de quínoa tostadas, nueces libres de agroquímicos, semillas de chía molidas, pasas de uva orgánica, azúcar integral orgánica tipo mascabo y canela. El producto fue comprado en un almacén de alimentos dietéticos de la Ciudad Autónoma de Buenos Aires, Argentina.

23: Hamburguesa de quínoa orgánica y algas Kelp. No declara ingredientes. El producto fue comprado en un almacén de alimentos dietéticos de la Ciudad Autónoma de Buenos Aires, Argentina.

24: Galletas de cereales inflados multicereal. Ingredientes: arroz integral, maíz pisingallo, sésamo integral, amaranto, sal marina. El producto fue comprado en un almacén de alimentos dietéticos de la Ciudad Autónoma de Buenos Aires, Argentina.

25: Galletas de cereal inflados multicereal sin sal. Ingredientes: arroz integral, maíz pisingallo, sésamo integral, semillas de amaranto. El producto fue comprado en un almacén de alimentos dietéticos de la Ciudad Autónoma de Buenos Aires, Argentina.

26: Galletas de cereales inflados multicereal dulces. Ingredientes: arroz integral, maíz pisingallo, sésamo integral, semillas de amaranto, edulcorantes: sacarina y ciclamato de sodio. Declara sin TACC. El producto fue comprado en un almacén de alimentos dietéticos de la Ciudad Autónoma de Buenos Aires, Argentina.

27: Barra de cereal con frutillas y yogur. Ingredientes: jarabe de glucosa, baño de moldeo blanco (azúcar impalpable, aceite vegetal, leche en polvo), crispines de arroz y maíz, azúcar orgánica, copos de arroz, aceite de girasol con omega 9, amaranto expandido, salvado de maíz, sorbitol, frutillas liofilizadas, yogur en polvo natural, jugo de frutilla en polvo, estabilizante (INS 412), emulsionante (INS 471) aroma idéntico al natural de frutilla. Declara "sin TACC". El producto fue comprado en un almacén de alimentos dietéticos de la Ciudad Autónoma de Buenos Aires, Argentina.

28: Barra de cereal con yogur sabor manzana verde. Ingredientes: jarabe de glucosa, baño de yogur (azúcar impalpable, aceite vegetal, leche en polvo, yogur en polvo, saborizantes y colorantes permitidos), crispines de arroz, azúcar orgánica, arroz crocante, manzana verde deshidratada, aceite de girasol con omega 9, amaranto expandido, semillas de chía, sorbitol, goma arábiga, salvado de maíz, emulsionante (INS 471), ácido cítrico, aromatizante permitido. Declara "sin TACC" y "no contiene gluten". El producto fue comprado en un almacén de alimentos dietéticos de la Ciudad Autónoma de Buenos Aires, Argentina.

29: Barrita de amaranto con semillas de chía. Ingredientes: amaranto inflado, chía molida, girasol orgánico, azúcar integral orgánica tipo mascabo y miel orgánica. 30: Barra de cereal con yogur sabor frutos del bosque. Ingredientes: jarabe de glucosa, baño de yogur (azúcar impalpable, aceite vegetal "low trans", leche en polvo, yogur en polvo, saborizantes y colorantes naturales permitidos), crispines de arroz, azúcar orgánica, arroz crocante, frutos del bosque deshidratados (frutillas, frambuesas y mora), aceite de girasol con omega 9, amaranto expandido, semillas de chía, sorbitol, goma arábiga, salvado de maíz, emulsionante: INS 471, ácido cítrico, aromatizante permitido. Declara "no contiene gluten". El producto fue comprado en un almacén de alimentos dietéticos de la Ciudad Autónoma de Buenos Aires, Argentina.

31: Barra de cereales con baño de yogur sabor frutilla. Ingredientes: crispines de arroz, jarabe de glucosa, baño de yogur, azúcar orgánica, arroz crocante, aceite de girasol de alto oleico, amaranto expandido, frutillas deshidratadas, sorbitol, goma arábiga, salvado de maíz, aromatizantes, emulsionante: INS 471, ácido cítrico. Declara "no contiene gluten”. El producto fue comprado en un almacén de alimentos dietéticos de la Ciudad Autónoma de Buenos Aires, Argentina.

32: Barra de cereal sabor frutilla con amaranto y semillas de chía. Ingredientes: arroz crocante, glucosa, baño de repostería, azúcar, amaranto expandido, aceite alto oleico de girasol, frutilla liofilizada, semillas de chía, salvado de maíz, sorbitol, goma arábiga, monoglicéridos de ácidos grasos, esencia de frutilla, ácido cítrico. El producto fue comprado en un almacén de alimentos dietéticos de la Ciudad 
Autónoma de Buenos Aires, Argentina.

33: Barra de cereal chocolate con amaranto y semillas de sésamo. Ingredientes: arroz crocante, glucosa, baño de repostería, azúcar, amaranto expandido, semillas de sésamo (ajonjolí), aceite alto oleico de girasol, cacao en polvo, salvado de maíz, sorbitol, goma arábiga, esencia de chocolate, monoglicéridos de ácidos grasos. El producto fue comprado en un almacén de alimentos dietéticos de la Ciudad Autónoma de Buenos Aires, Argentina.

34: Barra de cereales con manzana, amaranto y semillas de chía. Ingredientes: arroz crocante, glucosa, baño de repostería, azúcar, amaranto expandido, aceite alto oleico de girasol, manzana verde deshidratada, semillas de chía, salvado de maíz, sorbitol, goma arábiga, monoglicéridos de ácidos grasos. El producto fue comprado en un almacén de alimentos dietéticos de la Ciudad Autónoma de Buenos Aires, Argentina.

35: Barrita de amaranto con algarroba y chía. Ingredientes: amaranto inflado, semillas de girasol orgánico, semillas de sésamo molido orgánico, semillas de chía molidas, harina de algarroba, azúcar integral orgánico tipo mascabo, miel orgánica, extracto natural de vainilla. El producto fue comprado en un almacén de alimentos dietéticos de la Ciudad Autónoma de Buenos Aires, Argentina.

36: Barra de cereal con baño de chocolate. Ingredientes: crispines de arroz, jarabe de glucosa, baño de cacao, azúcar orgánica, arroz crocante, aceite de girasol alto oleico, amaranto expandido, cacao en polvo, harina de soja, sorbitol, goma arábiga, semillas de chía, salvado de maíz, aromatizante, emulsionante: INS 471, ácido cítrico. Declara "no contiene gluten". El producto fue comprado en un almacén de alimentos dietéticos de la Ciudad Autónoma de Buenos Aires, Argentina.

37: Barra de cereal con yogur sabor durazno. Ingredientes: jarabe de glucosa, baño de yogur (azúcar impalpable, aceite vegetal "low trans", leche en polvo, yogur en polvo, saborizantes y colorantes naturales permitidos), crispines de arroz, azúcar orgánica, arroz crocante, duraznos deshidratados, aceite de girasol con omega 9, amaranto expandido, semillas de chía, sorbitol, goma arábiga, salvado de maíz, emulsionante: INS 471, ácido cítrico, aromatizante permitido. Declara "no contiene gluten". El producto fue comprado en un almacén de alimentos dietéticos de la Ciudad Autónoma de Buenos Aires, Argentina.

Las muestras $2-14$ y $18-37$ corresponden a productos comerciales.

\section{MÉTODOS \\ Muestreo}

Para los alimentos en polvo, semillas y hojuelas se realizó un muestreo de tipo estratificado (muestras 1-22). En los productos que se expendían con varias unidades dentro de su envase original se efectuó un muestreo aleatorio (muestras 23-26). Se tomó la totalidad del contenido del paquete para su procesamiento en aquellas muestras donde el envase contenía sólo una unidad (muestras 27-37).

\section{Procesamiento de las muestras}

La totalidad de las muestras fueron homogeneizadas en molino de cuchillas marca Retsch ${ }^{\circledR}$ modelo Grindomix GM200 hasta una granulometría de 35 mesh.

\section{Método de enzimoinmunoensayo}

Se utilizó un kit comercial (RIDASCREEN® gliadin, art. ${ }^{\circ}$ R7001) de R-Biopharm (6). El mismo consiste en un enzimoinmunoensayo sandwich para el análisis cuantitativo de prolaminas de trigo (gliadinas), centeno (secalinas) y cebada (hordeínas).

Se pesaron $0,25 \mathrm{~g}$ de cada muestra homogeneizada y se extrajo con $2,5 \mathrm{~mL}$ de solución cóctel (RIDASCREEN@ Gliadin, art. $\mathrm{N}^{\circ} \mathrm{R} 7006$ ) durante $40 \mathrm{~min}$ a $50^{\circ} \mathrm{C}$ en un baño termostatizado con agitación. Se dejó enfriar y se agregaron $7,5 \mathrm{~mL}$ de etanol $80 \%$. Se mezcló durante $1 \mathrm{~h}$ en posición horizontal a temperatura ambiente. Se centrífugó a temperatura ambiente durante 10 min a 2900 rpm. Se trasvasó cada sobrenadante a tubos eppendorf, que se conservaron hasta el momento del ensayo. Las muestras se diluyeron de acuerdo con las instrucciones del kit (6)

La solución de extracción utilizada, solución cóctel (RIDASCREEN ${ }^{\circledR}$ Gliadin, Art. N ${ }^{\circ}$ R7006), contiene clorhidrato de guanidina (agente desagregante) y 2-mercaptoetanol (agente reductor). Esta solución fue diseñada especialmente para alimentos "sin gluten" procesados a altas temperaturas. El sistema permite obtener rendimientos del $100 \%$ de gluten, tanto en alimentos procesados a altas temperaturas como en no procesados por calor, en contraste con los bajos rendimientos (10 - $30 \%$ ) del sistema convencional de extracción con etanol/agua. (7).

Todas las muestras se extrajeron por duplicado y cada extracto se analizó por enzimoinmunoensayo por duplicado. El procedimiento de análisis se realizó siguiendo las instrucciones del kit (6). La lectura de la absorbancia de cada uno de los pocillos de la placa de ELISA se realizó a $450 \mathrm{~nm}$ con un lector de microplaca Rayto ${ }^{\circledR}$ RT-2100C. Se realizó una corrección de fondo leyendo además a $600 \mathrm{~nm}$. 
Los resultados de cada muestra analizada (expresada en $\mathrm{mg} / \mathrm{Kg}$ de gliadina) se obtuvieron utilizando el programa Ridasoft win de R-Biopharm. De acuerdo a las especificaciones del kit (6), el contenido de gluten $(\mathrm{mg} / \mathrm{Kg})$ corresponde al contenido de gliadina $(\mathrm{mg} / \mathrm{Kg})$ multiplicado por 2.

\section{RESULTADOS Y DISCUSIÓN}

En Argentina, el Código Alimentario Argentino (CAA) considera dentro del Capítulo XVII, Alimentos dietéticos o alimentos para regímenes especiales, a los alimentos libres de gluten, sin TACC (8). Entre las normas generales para alimentos dietéticos se establece que "deben ser acondicionados en su lugar de elaboración o en otras plantas de la misma empresa y que se deben expender en envases que satisfagan las exigencias del CAA. Está prohibido el fraccionamiento y expendio a granel o al detalle". Además, establece que "las empresas elaboradoras deben contar con un director técnico". En particular, para los alimentos libres de gluten, sin TACC, especifica en el artículo 1382 bis que "son aquellos que están preparados con ingredientes que por su origen natural y por la aplicación de buenas prácticas de elaboración, que impidan la contaminación cruzada, no contienen prolaminas de todas las especies de Triticum, de trigo duro, centeno, cebada, avena ni de sus variedades cruzadas. Estos productos se rotularán con la denominación del producto que se trate seguido de la indicación "libre de gluten" debiendo incluir además la leyenda "Sin TACC" en las proximidades de la denominación del producto con caracteres de buen realce, tamaño y visibilidad.

Para comprobar la condición libre de gluten deben utilizarse aquellas técnicas que la Autoridad Sanitaria Nacional evalúe y acepte. En el artículo 1383 establece que para la aprobación de los alimentos libres de gluten, los elaboradores y/o importadores deberán presentar ante la Autoridad Sanitaria de su jurisdicción un análisis que avale la condición de "libre de gluten" otorgado por un organismo oficial o entidad con reconocimiento oficial según lo establecido en el artículo 1382 y un programa de buenas prácticas de fabricación, con el fin de asegurar la no contaminación con derivados de trigo, avena, cebada y centeno en los procesos, desde la recepción de las materias primas hasta la comercialización del producto final".

En los artículos mencionados no se hace referencia a valores límites para considerar a un alimento como libre de gluten.

Dado que el Codex Alimentarius (4) considera a un alimento como exento de gluten cuando contiene menos de $20 \mathrm{mg} / \mathrm{kg}$ de gluten, en el presente trabajo se adoptó este criterio como valor de corte.

En la tabla 1 se presenta el contenido de gluten de las muestras analizadas.

Nueve de los productos evaluados superaron ese límite (24\%): tres barras de cereales que declaraban "sin TACC" y/o "no contiene gluten" (muestras 27, 28 y 31 ), dos barras de cereales que no tenían ninguna declaración respecto del contenido de gluten (muestras 29 y 35), una muestra de harina de amaranto envasada que declaraba "apto para celíacos" (muestra 2), una muestra de semillas de quínoa comprada al detalle en un mercado de la ciudad de Salta, (muestra 7), una mezcla de semillas molidas (muestra 11) y una muestra de amaranto popeado comprado suelto en un almacén de alimentos dietéticos (muestra 13).

Las cuatro muestras que declaraban "sin TACC" y/o "no contiene gluten" o "apto para celíacos", no presentaban en la denominación del producto la leyenda libre de gluten (muestras 2, 27, 28 y 31). Esto nos indica que el alimento no había sido registrado como alimento dietético o para regímenes especiales libre de gluten como lo especifica el artículo 1382 bis del Capítulo XVII del CAA (8).

En nuestro país, un gran número de productos comercializados como libres de gluten no sólo no fueron aprobados como tales, sino que, además, luego de su inscripción, han modificado su rótulo e incluido el símbolo internacional de "libre de gluten" o el de "Sin TACC", o leyendas como "apto para celíacos", "no contiene gluten" o "sin TACC", ninguno de ellos autorizado por la normativa vigente. Los resultados positivos hallados en el presente trabajo en las muestras 2 , 27,28 y 31 demuestran la importancia de que este tipo de alimentos sean registrados y controlados de acuerdo con la normativa vigente (8).

En las veintiocho muestras restantes $(76 \%)$ se evidenció un contenido de gluten inferior a los $20 \mathrm{mg} /$ $\mathrm{Kg}$.

Las nueve muestras que dieron resultado positivo (muestras 2, 7, 11, 13, 27, 28, 29, 31 y 35) se estima que tuvieron una contaminación cruzada en su proceso de elaboración. Se debe tener en cuenta que, si bien la muestra 11 declara en su lista de ingredientes semillas de avena molidas, el resultado positivo de gluten no se debe a este ingrediente ya que el kit no detecta proteínas de avena (6) lo que indicaría probablemente una contaminación cruzada con gliadinas de trigo. Las muestras 7 (semillas de quínoa) y 13 (amaranto popeado) fueron compradas al detalle en un almacén de alimentos dietéticos. La contaminación de estas muestras probablemente se debió a su forma de expendio que favorece la posible contaminación con otros productos del comercio que 


\section{TABLA 1}

Contenido de gluten en alimentos elaborados con amaranto, quínoa y/o chía (mg/kg).

Muestra $\mathrm{N}^{\circ} \quad$ Contenido de gluten $(\mathrm{mg} / \mathrm{Kg})$

$\begin{array}{lc}1 & 1 \\ 2 & 34 \\ 3 & 9 \\ 4 & 12 \\ 5 & 10\end{array}$


pueden contener gluten; ya que en los mismos se utilizan para dispensarlos elementos (cucharas, cucharines, entre otros) que se comparten sin ningún tratamiento posterior para eliminar contaminaciones cruzadas. La muestra 2 (harina de amaranto envasada) se pudo haber contaminado en su proceso de obtención (molienda) y/o en su fraccionamiento.

De acuerdo con los resultados obtenidos en el presente trabajo, es importante que los alimentos libres de gluten, sin TACC, se expendan envasados desde su lugar de origen y que los fabricantes de estos alimentos y los directores técnicos de estas empresas se comprometan a controlar, en todas las etapas de elaboración, que no ocurra una contaminación cruzada que pueda alterar la condición del alimento de ser libre de gluten, $\sin$ TACC.

\section{CONCLUSIÓN}

Los hallazgos del presente trabajo indican que los productos elaborados con amaranto, quínoa y/o chía son seguros para personas con celiaquía. Sin embargo, de acuerdo con nuestros resultados, los fabricantes deben implementar buenas prácticas de manufactura en las diferentes etapas de elaboración de alimentos libres de gluten, sin TACC y las personas celíacas no deben consumir alimentos supuestamente aptos, que se expenden al detalle, por la posible contaminación cruzada que puede darse en los comercios.

\section{RESUMEN}

Amaranto, quínoa y chía, por ser naturalmente libres de gluten, pueden ser incorporados en la dieta celíaca. Con el objeto de evaluar una posible contaminación cruzada con gliadinas no permitidas, se analizaron 37 alimentos con estos ingredientes mediante un enzimoinmunoensayo utilizando RIDASCREEN gliadin de R-Biopharm. Considerando el contenido máximo de gluten establecido por el Codex Alimentarius (20 mg/ $\mathrm{Kg}$ ), nueve productos superaron la norma: tres barras de cereales que declaraban "sin TACC" y/o "no contiene gluten", dos barras de cereales que no tenían ninguna declaración respecto del contenido de gluten, una mezcla de semillas molidas, una muestra de amaranto popeado comprado al detalle, una muestra de semillas de quinoa comprada suelta en un mercado de la provincia de Salta y una muestra de harina de amaranto envasada que declaraba "apto para celíacos". En las veintiocho muestras restantes se evidenció un contenido de gluten inferior a los $20 \mathrm{mg} / \mathrm{Kg}$. Los productos elaborados con amaranto, quínoa y/o chía son seguros para personas con celiaquía; sin embargo, los fabricantes deben implementar buenas prácticas de manufactura en las diferentes etapas de ela- boración de alimentos libres de gluten y las personas celíacas no deben consumir alimentos supuestamente aptos que se expenden al detalle, por la posible contaminación cruzada que puede darse en los comercios.

Palabras clave: amaranto; quínoa; chía; gliadina; celiaquía.

Dirigir la correspondencia a:

Profesora

Laura Beatriz López

Cátedras de Bromatología y Nutrición

Facultad de Farmacia y Bioquímica

Universidad de Buenos Aires

Junin 956, Piso 2

Buenos Aires. Argentina.

E-mail: laulop@ffyb.uba.ar

Agradecimientos: Parcialmente financiado por UBACYT B 081 y B 098.

\section{BIBLIOGRAFÍA}

1. Cueto Rúa EA, Nanfito G. Enfermedad Celíaca. Nestlé Nutrición. Octubre 2002; 6 (6): 4-13.

2. Cueto Rúa EA. Celiaquía, un modo de ser. Parábola Editorial, Ciudad Autónoma de Buenos Aires, 2007.

3. Green P and Cellier C. 2007. Celiac Disease. N. Engl. J. Med. 357:17. 1731 - 1743.

4. Codex Alimentarius, 2009. CODEX STAN 118 1979, enmendado 1983, revisado 2008. Disponible en: http://www.codexalimentarius.net/web/more_ info.jsp?id_sta=291. Consultado: noviembre de 2009.

5. Itúrbide G, Gispert M. Cultivos marginados: otra perspectiva de 1492. FAO, Roma, 1992.

6. R-Biopharm, 2008. Disponible en: http://w ww.rbiopharm.com/product_site.php?product_id $=252 \&$ product_class_one=QWxsZXJnZW5z\&product_ class_two $=$ R $2 \times p Y W R p b g==\&$ product_class _three=\&product_class_four=\&product_range= Food $\% 20$ and $\% 20$ Feed $\% 20$ Analysisç. Consultado: noviembre de 2009.

7. Méndez E. 2001. Problemas generales relacionados con la analítica del gluten (II). Revista Transferencia de Tecnología. Revista 4. Disponible en: http:// www.madrimasd.org/revista/revista4/transferencia/ transf erencias.asp. Consultado: noviembre de 2009.

8. Código Alimentario Argentino, actualizado, 2009. Disponible en: http://www.anmat.gov.ar/codigoa/ CAPITULO_XVII_Dieteticos_actualiz-06-09.pdf. Consultado: noviembre de 2009. 